\title{
The Impact of Text Message On Self-Management for Coronary Heart Disease: A Meta-Analysis of Randomized Controlled Trials
}

\author{
Yong Wan, Xin $\mathrm{Wu}$, Yan Kou \\ Special Survey Dept.(ECG room), Sichuan Luzhou People’s Hospital, Luzhou City, Sichuan, P.R.China
}

\section{ABSTRACT}

Introduction: The influence of text message on selfmanagement for coronary heart disease remains controversial. We conducted a systematic review and meta-analysis to explore the impact of text message versus usual care on selfmanagement for coronary heart disease.

Methods: We searched PubMed, EMbase, Web of Science, EBSCO, and Cochrane library databases through July 2018 for randomized controlled trials (RCTs), assessing the effect of text message versus usual care on self-management for coronary heart disease. This meta-analysis is performed using the random-effect model.

Results: Six RCTs involving 1,158 patients are included in the meta-analysis. Overall, compared with a control group for coronary heart disease, text message intervention has no substantial influence on self-efficacy (Std. MD $=2.37 ; 95 \%$ $\mathrm{CI}=-2.61$ to $7.35 ; P=.35), \mathrm{LDL}($ Std. $\mathrm{MD}=-1.81 ; 95 \%$ $\mathrm{CI}=-4.80$ to $1.18 ; P=.24), \mathrm{HDL}(\mathrm{Std} . \mathrm{MD}=-1.15 ; 95 \%$ $\mathrm{CI}=-2.83$ to $0.54 ; P=.18)$, BMI (Std. $\mathrm{MD}=-3.61 ; 95 \% \mathrm{CI}$ $=-9.48$ to $2.26 ; P=.23)$, systolic blood pressure (Std. MD $=-3.46 ; 95 \% \mathrm{CI}=-9.03$ to $2.12 ; P=.22)$, diastolic blood pressure (Std. $\mathrm{MD}=-2.03 ; 95 \% \mathrm{CI}=-5.90$ to $1.85 ; P=.31$, non-smoker $(\mathrm{RR}=1.12 ; 95 \% \mathrm{CI}=0.78$ to $1.62 ; P=.53)$, and physical activity $(\mathrm{RR}=1.57 ; 95 \% \mathrm{CI}=0.63$ to $3.90 ; P=.33)$.

Conclusions: Text message intervention demonstrates no positive impact on self-efficacy, treatment adherence, and the control of risk factors in patients with coronary heart disease.

\section{INTRODUCTION}

Coronary heart diseases are the most common cause of morbidity and mortality among cardiovascular diseases [Sayols-Baixeras 2014; John 2018; Mahonen 2000]. Their incidence rate is 181.4 in every 100,000 individuals, with $46 \%$ of mortality cases [Boroumand 2016]. Patients with coronary heart diseases subsequently may suffer from congestive heart failure, cardiac arrhythmias and cardiogenic shock, and require much expense for care and treatment [Brainin 2018; Feng 2018]. The ideal treatments for coronary heart diseases

Received April 17, 2019; accepted October 23, 2019.

Correspondence: Yan Kou, 1 Zhongxiao Road, Fiangyang District, Luzhou City, Sichuan Province, China; 021-63501832; fax: 021-63501832 (e-mail:905135782@qq.com). include pharmaceutical, interventional, and surgical measures, management of risk factors, and lifestyle modifications [Bitton 2013; Mohr 2013; Janssen 2013].

Traditional follow-up methods include patients' referral to care centers in person and home visits by health care personnel which require human resources, time, and high expenses [Boroumand 2016]. An efficient, easy, and cost-effective method of telenursing is through text message and telephone [Eng 2014]. Text message is a new, effective, and cost-effective communication method to follow patient status and transference of health information possible in the most remote areas [Boroumand 2016]. Telephone follow-up has emerged as one of the most cost-effective and efficient methods of follow-up in chronic diseases and reduces unnecessary patient visits.

Cognitive-behavioral factors such as self-efficacy can be changed in the promotion of health through lifestyle modification interventions in patients with coronary heart diseases [Cataldo 2013]. Self-efficacy is defined as the individuals' understanding of their own ability to change or continue a behavior successfully [Keefer 2011]. Improved self-efficacy is effective to promote treatment adherence and healthy behaviors of patients [Kang 2013]. The efficacy of text message to improve self-efficacy and other benefits for coronary heart disease has not been well established. Recently, several studies on the topic have been published, and the results have been

Potentially relevant studies in the first search $\mathrm{n}=542$

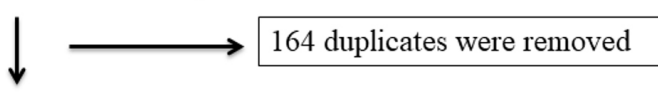

378 initial included

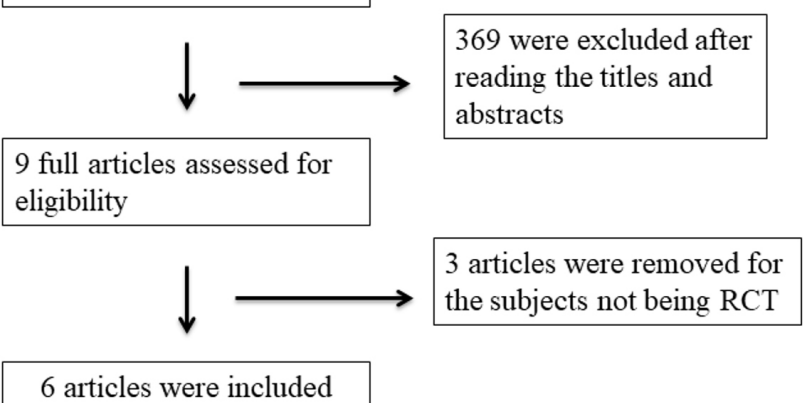

6 articles were included

Figure 1. Flow diagram of study searching and selection process. 


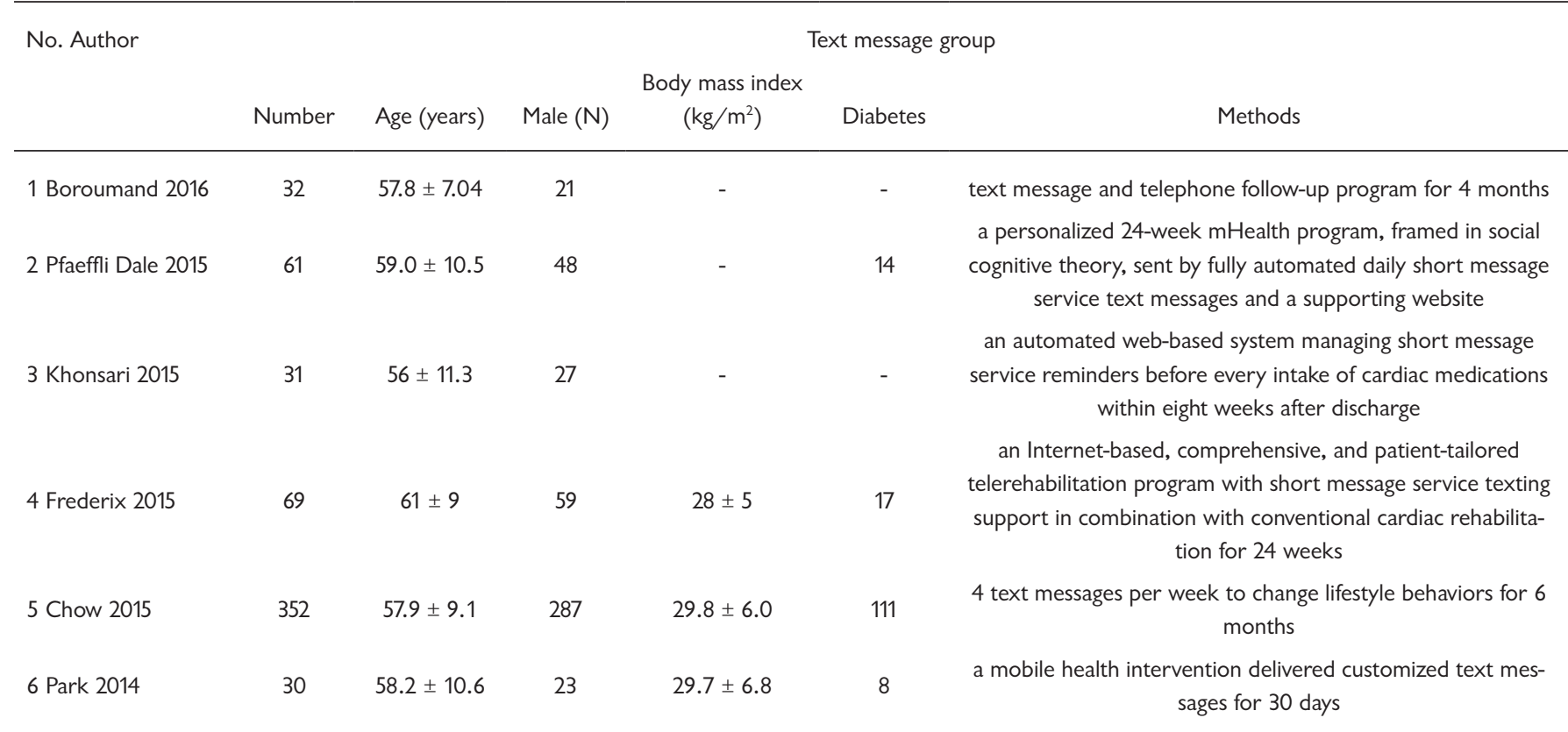

conflicting [Boroumand 2016; Pfaeffli 2015; Khonsari 2015; Frederix 2015]. With accumulating evidence, we therefore perform a systematic review and meta-analysis of RCTs to investigate the efficacy of text message versus usual care for coronary heart disease.

\section{MATERIALS AND METHODS}

Ethical approval and patient consent are not required because this is a systematic review and meta-analysis of previously published studies. The systematic review and metaanalysis are conducted and reported in adherence to PRISMA (Preferred Reporting Items for Systematic Reviews and Meta-Analyses) [Moher 2009].

Search strategy and study selection: Two investigators independently searched the following databases (inception to July 2018): PubMed, EMbase, Web of Science, EBSCO, and Cochrane library databases. The electronic search strategy was conducted using the following keywords: 'text message' and 'coronary disease.' We also checked the reference lists of the screened full-text studies to identify other potentially eligible trials.

The inclusive selection criteria were as follows: (i) population: patients with coronary heart disease; (ii) intervention: text message; (iii) comparison: usual care; and (iv) study design: RCT.

Data extraction and outcome measures: We extracted the following information: author, number of patients, age, male, body mass index (BMI), diabetes, and detail methods in each group etc. Data independently was extracted by two investigators, and discrepancies were resolved by consensus. We also contacted the corresponding author to obtain the data, when necessary. No simplifications and assumptions are made.
The primary outcome is self efficacy. Secondary outcomes include low-density lipoprotein (LDL), high-density lipoprotein (HDL), BMI, systolic blood pressure, diastolic blood pressure, non-smoker, and physical activity.

Quality assessment in individual studies: Methodological quality of the included studies was independently evaluated using the modified Jadad scale [Jadad 1996]. There are 3 items for Jadad scale: randomization (0-2 points), blinding ( $0-2$ points), dropouts and withdrawals ( $0-1$ points). The score of Jadad Scale varies from 0 to 5 points. An article with Jadad score $\leq 2$ is considered to be of low quality. If the Jadad score $\geq 3$, the study is thought to be of high quality [Kjaergard 2001].

\section{STATISTICAL ANALYSIS}

We estimate the standard mean difference (Std. MD) with $95 \%$ confidence interval (CI) for continuous outcomes (self efficacy, LDL, HDL, BMI, systolic blood pressure, and diastolic blood pressure) and risk ratio (RR) with $95 \%$ CIs for dichotomous outcomes (non-smoker and physical activity). A random-effects model is used regardless of heterogeneity. Heterogeneity is reported using the I 2 statistic, and $\mathrm{I} 2>50 \%$ indicates significant heterogeneity [Higgins 2002]. Whenever significant heterogeneity is present, we search for potential sources of heterogeneity via omitting one study in turn for the meta-analysis or performing subgroup analysis. Publication bias is not evaluated because of the limited number $(<10)$ of included studies. All statistical analyses are performed using Review Manager Version 5.3 (The Cochrane Collaboration, Software Update, Oxford, UK). 


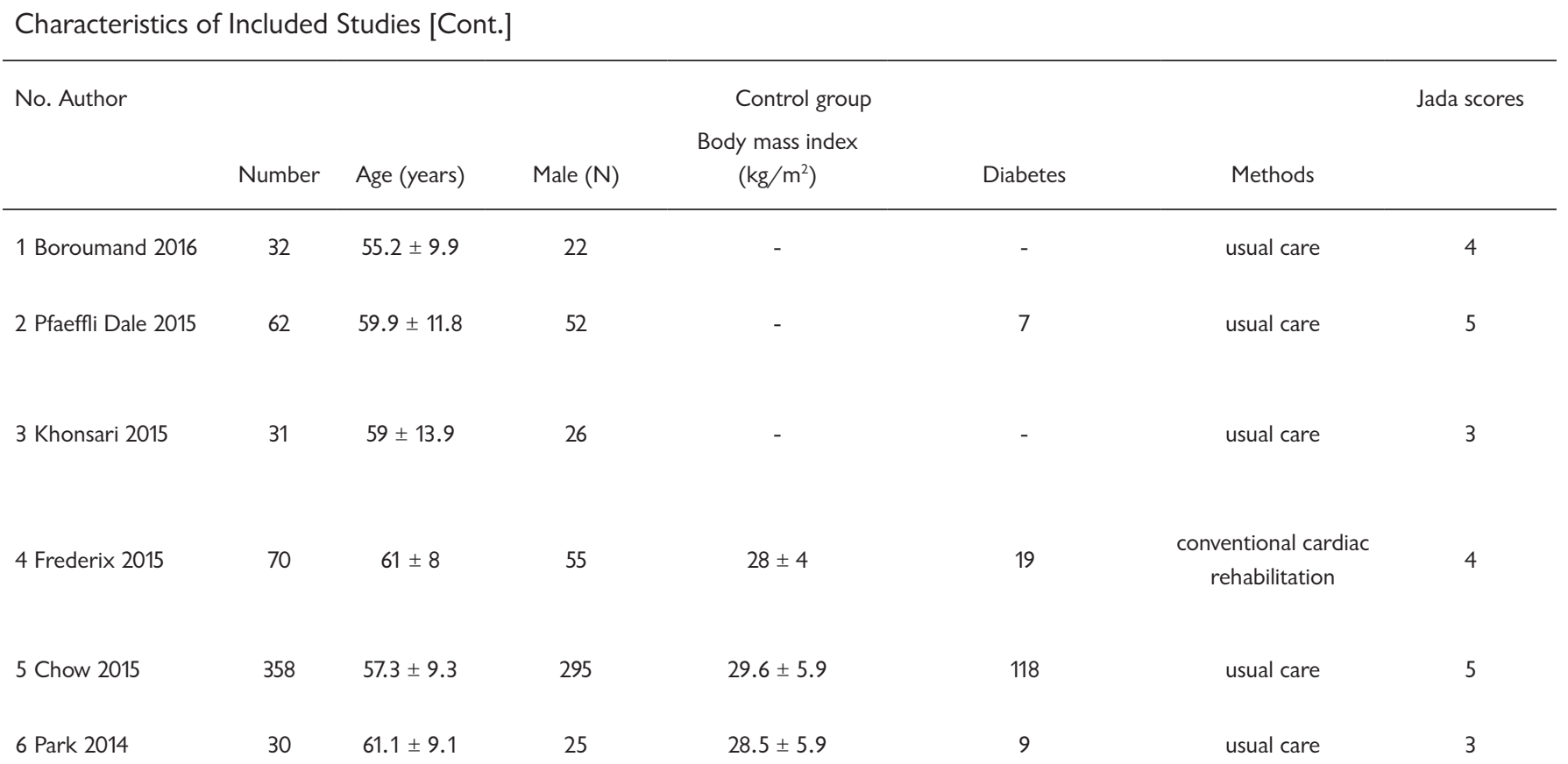

\section{RESULTS}

Literature search, study characteristics and quality assessment: A detailed flowchart of the search and selection results is shown in Figure 1. Potentially relevant are 542 articles that initially were identified. Finally, 6 RCTs that meet our inclusion criteria are included in the meta-analysis [Boroumand 2016; Pfaeffli 2015; Khonsari 2015; Frederix 2015; Chow 2015; Park 2014].

The baseline characteristics of the 6 eligible RCTs in the meta-analysis are summarized in Table. The 6 studies were published between 2014 and 2016, and sample sizes ranged from 62 to 710 with a total of 1,158 . Two RCTs report local injection of methylene blue after traumatic thoracolumbar fixation [Farrokhi 2016], and lumbar open discectomy [Farrokhi 2016]. The detail methods of text message are different in each RCT, and the duration ranges from 1 month to 6 months.

Among the 6 studies included here, 2 studies report self efficacy [Boroumand 2016; Pfaeffli 2015], 2 studies report LDL, HDL and BMI [Pfaeffli 2015, Chow 2015], 3 studies report systolic blood pressure and diastolic blood pressure [Pfaeffli 2015; Frederix 2015; Chow 2015], and 2 studies report non-smoker and physical activity [Pfaeffli 2015; Chow 2015]. Jadad scores of the 6 included studies vary from 4 to 5 , and all 6 studies are considered to be high-quality ones according to quality assessment.

Primary outcome: self efficacy. This outcome data is analyzed with the random-effects model, and the pooled estimate of the two included RCTs suggested that compared with the control group for coronary heart disease, text message is not associated with substantially improved self-efficacy (Std. $\mathrm{MD}=2.37 ; 95 \% \mathrm{CI}=-2.61$ to $7.35 ; P=.35$ ) with significant heterogeneity among the studies $\left(\mathrm{I}^{2}=99 \%\right.$, heterogeneity $P<.00001$ ) (Figure 2).

Sensitivity analysis: Significant heterogeneity is observed among the included studies for the self-efficacy, but there are just 2 RCTs included in the meta-analysis. Thus, we did not perform sensitivity analysis via omitting 1 study in turn or subgroup analysis to detect the heterogeneity.

Secondary outcomes: Compared with the control group for coronary heart disease, text message shows no significant impact on LDL (Std. MD $=-1.81 ; 95 \% \mathrm{CI}=-4.80$ to 1.18 ; $P=.24$; Figure 3), HDL (Std. MD $=-1.15 ; 95 \% \mathrm{CI}=-2.83$ to $0.54 ; P=.18$; Figure 4$), \mathrm{BMI}$ (Std. $\mathrm{MD}=-3.61 ; 95 \% \mathrm{CI}$ $=-9.48$ to $2.26 ; P=.23$; Figure 5$)$, systolic blood pressure (Std. $M D=-3.46 ; 95 \% C I=-9.03$ to $2.12 ; P=.22$; Figure 6 ), diastolic blood pressure (Std. $\mathrm{MD}=-2.03 ; 95 \% \mathrm{CI}=-5.90$ to $1.85 ; P=.31$; Figure 7$)$, non-smoker $(\mathrm{RR}=1.12 ; 95 \%$ $\mathrm{CI}=0.78$ to $1.62 ; P=.53$; Figure 8$)$, and physical activity (RR $=1.57 ; 95 \% \mathrm{CI}=0.63$ to $3.90 ; P=.33$; Figure 9$)$.

\section{DISCUSSION}

Mobile phone text message-based interventions have been reported to be a potential means of modifying health behaviors [Fjeldsoe 2009]. Some studies reveal its efficacy to change individual health behaviors of smoking, weight loss, and physical activity to improve medical management of diabetes, and medication adherence [Franklin 2006]. Text messaging delivering via mobile phone intervention has particular value and obtains wide population effects, without the requirement of a smartphone. Health and mobile health interventions have easy scalability owing to affordability and ability to deliver personalized services [Lester 2010; Franklin 2015]. 


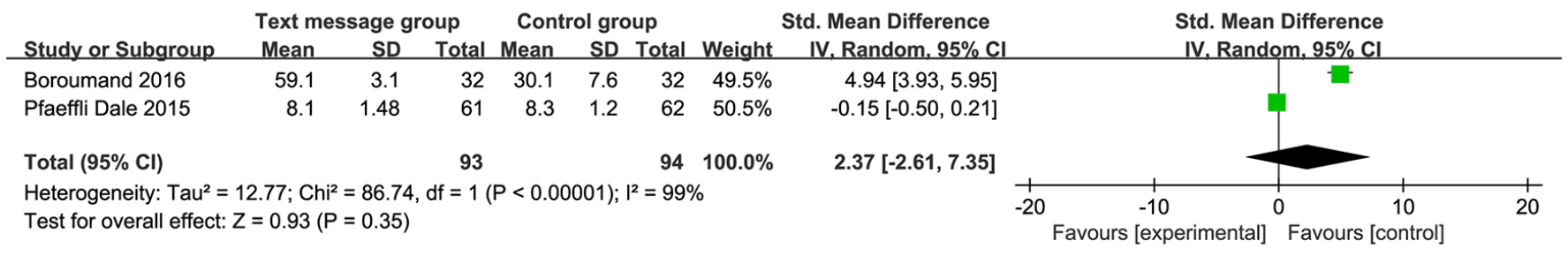

Figure 2. Forest plot for the meta-analysis of self efficacy.

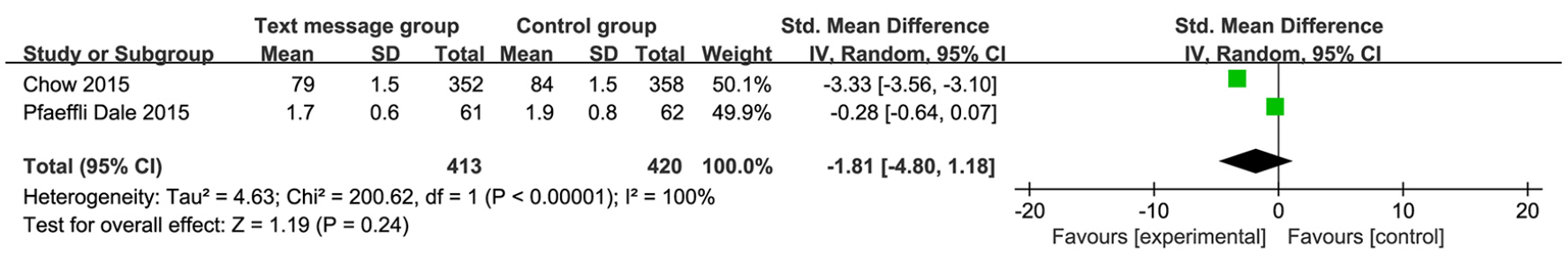

Figure 3. Forest plot for the meta-analysis of low-density lipoprotein (LDL).

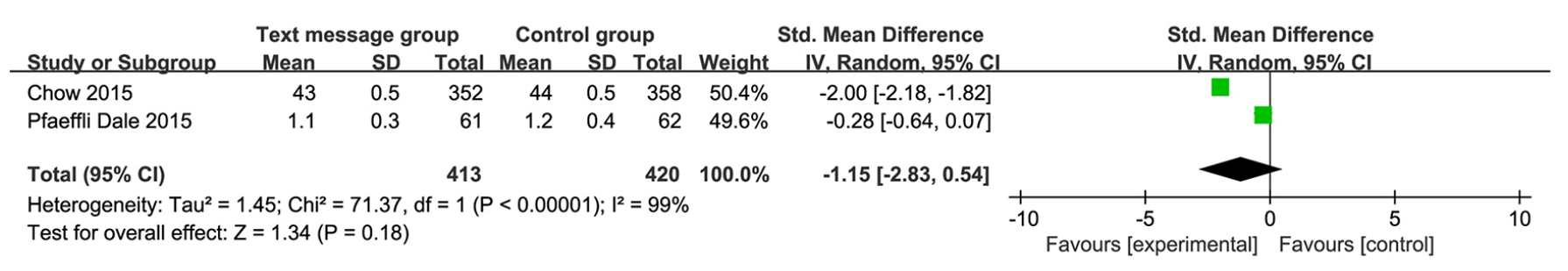

Figure 4. Forest plot for the meta-analysis of high-density lipoprotein (HDL).

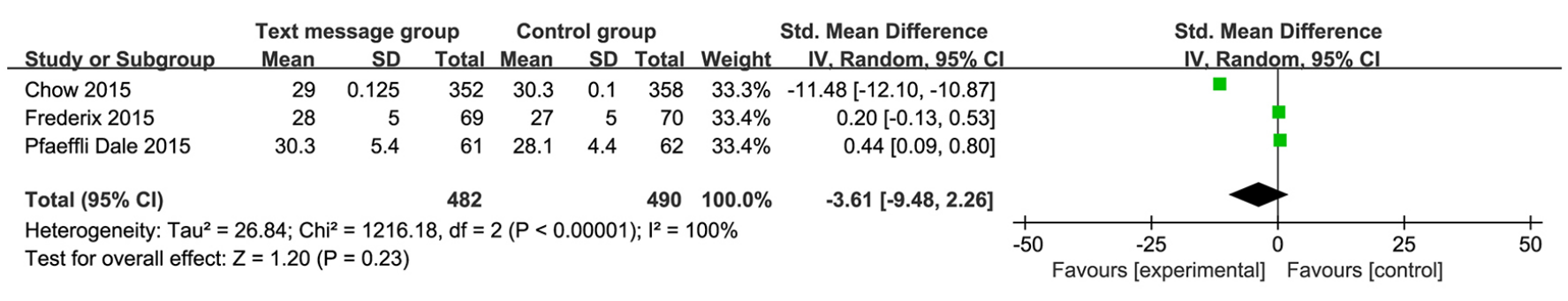

Figure 5. Forest plot for the meta-analysis of BMI.

Many clinical trials have shown the effectiveness of mobile phone text messaging to promote weight loss, physical activity, glycemic control in diabetes, and medication adherence [Franklin 2006; Lester 2010; Patrick 2009; Hurling 2007]. In one RCT involving 70 patients with coronary heart disease, cardiac self-efficacy score significantly is increased after 3 and 4 months of text message intervention [Boroumand 2016]. One study exploring the effect of a text message-based weight loss program on weight loss and dietary habits of overweight individuals finds that text message intervention has a greater reduction in weight and improvement in dietary habits compared with a control group [Norman 2013]. Text message intervention provides important benefits for physical activity self-efficacy, and frequency and duration of physical activity 6 weeks and 6 months after the intervention [Furber 2010].

However, in another study, text message and telephone follow-up of cardiac patients with diabetes shows no significant difference of self-efficacy and self-care behavior between the intervention and control groups [Wu 2012]. One study aims to explore the efficacy of a text message intervention on medication adherence in patients with coronary heart disease, and the results reveal no significant difference between 


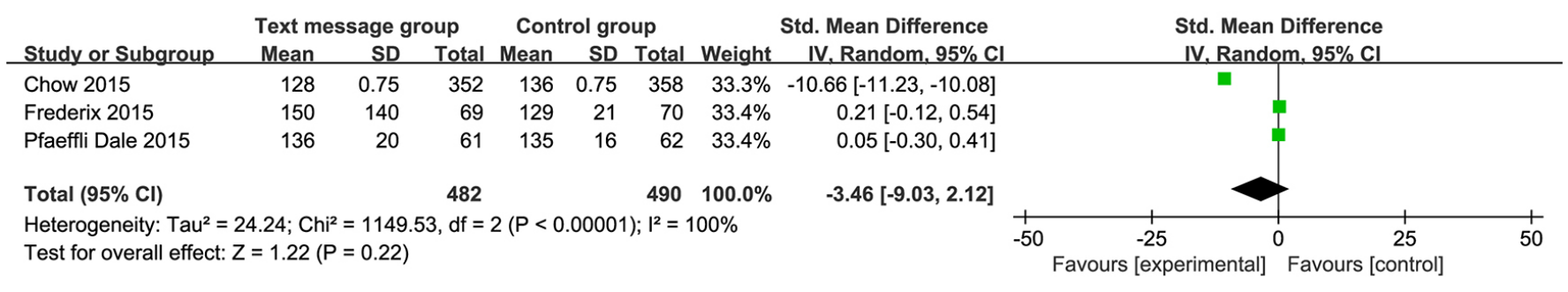

Figure 6. Forest plot for the meta-analysis of systolic blood pressure.

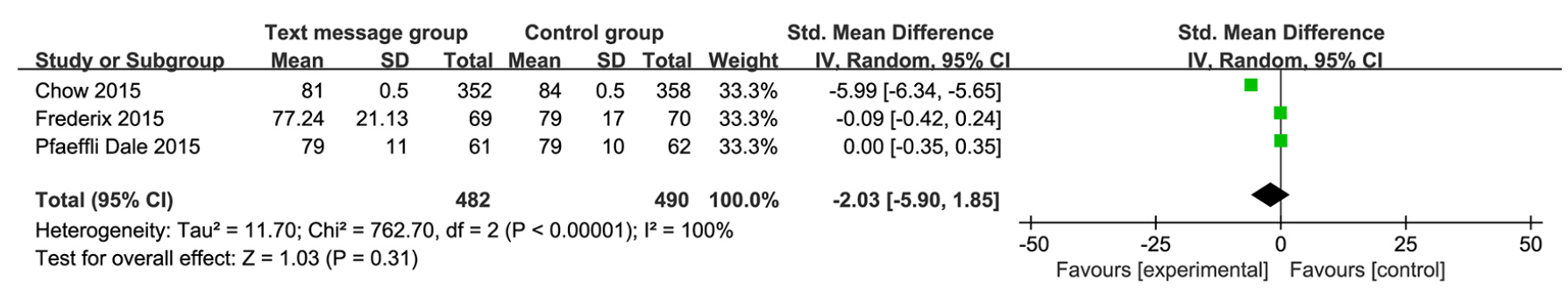

Figure 7. Forest plot for the meta-analysis of diastolic blood pressure.

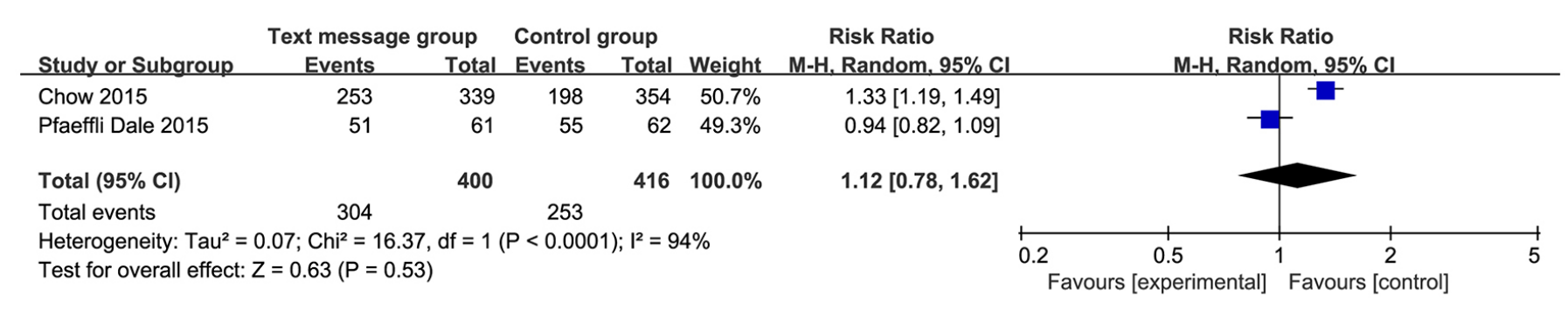

Figure 8. Forest plot for the meta-analysis of non-smoker.

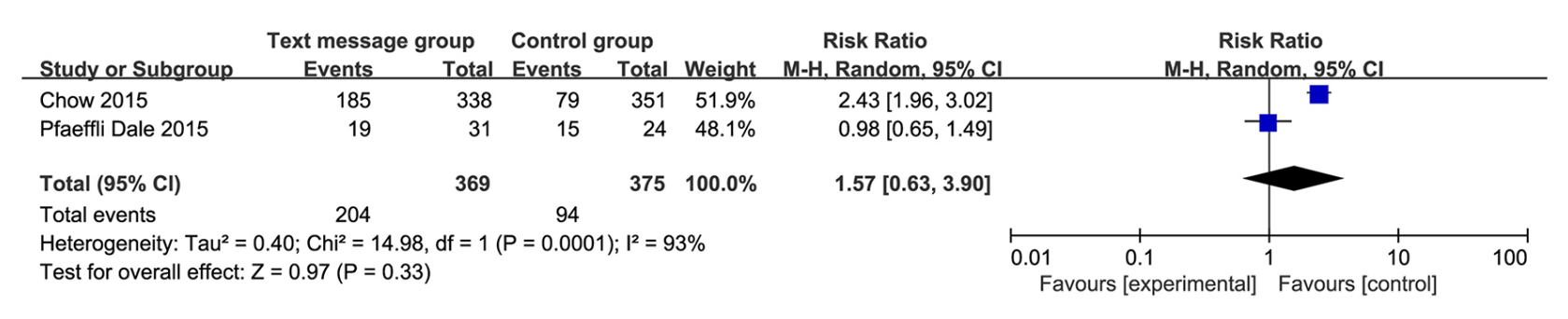

Figure 9. Forest plot for the meta-analysis of physical activity.

text message intervention and control group in terms of selfefficacy in correct use of medication dose [Park 2014].

Our meta-analysis suggests that text message shows no additional benefits of self-efficacy, the number of non-smoker, and physical activity compared with the control group for coronary heart disease. The risk factors for coronary heart disease are evaluated by LDL and HDL level, BMI, systolic and diastolic blood pressure. In our meta-analysis, there is no significant difference of these indexes between the two groups. Regarding the sensitivity, there is significant heterogeneity.
Several reasons may explain it. First, different evaluation score systems are applied for the same index, such as self efficacy [Boroumand 2016; Pfaeffli 2015]. Second, detail methods of text message are various in each RCT. Third, the duration of text message ranges from 30 days to 6 months, which may have some impact on the pooling results.

This meta-analysis has several potential limitations that should be taken into account. First, our analysis is based on only 6 RCTs and 3 of them have a relatively small sample size $(\mathrm{N}<100)$. More RCTs with large samples should be conducted 
to explore this issue. There is significant heterogeneity, different methods and duration of text message intervention, as well as evaluation score systems in included RCTs may have an influence on the pooling results. Finally, some unpublished and missing data might lead bias to the pooled effect.

\section{CONCLUSION}

Text message intervention has no remarkable beneficial effects on coronary heart disease.

\section{REFERENCES}

Bitton A, Choudhry NK, Matlin OS, Swanton K, Shrank WH. 2013. The impact of medication adherence on coronary artery disease costs and outcomes: a systematic review. The American journal of medicine. $126: 357$ e $7-$ e 27.

Boroumand S, Moeini M. 2016. The effect of a text message and telephone follow-up program on cardiac self-efficacy of patients with coronary artery disease: A randomized controlled trial. Iranian journal of nursing and midwifery research. 21:171-6.

Brainin P, Hoffmann S, Fritz-Hansen T, Olsen FJ, Jensen JS, BieringSorensen T. 2018. Usefulness of Postsystolic Shortening to Diagnose Coronary Artery Disease and Predict Future Cardiovascular Events in Stable Angina Pectoris. Journal of the American Society of Echocardiography: official publication of the American Society of Echocardiography. 31:870-9 e3.

Cataldo R, John J, Chandran L, Pati S, Shroyer AL. 2013. Impact of physical activity intervention programs on self-efficacy in youths: a systematic review. ISRN obesity. 2013:586497.

Chow CK, Redfern J, Hillis GS, Thakkar J, Santo K, Hackett ML, et al. 2015. Effect of Lifestyle-Focused Text Messaging on Risk Factor Modification in Patients With Coronary Heart Disease: A Randomized Clinical Trial. Jama. 314:1255-63.

Eng JA, Hunter CJ, Handley MA, Boscardin CK, Gonzales R, Ackerman SL. 2014. Patient attitudes about specialty follow-up care by telephone. The American journal of managed care. 20:164-7.

Farrokhi MR, Lotfi M, Masoudi MS, Gholami M. 2016. Effects of methylene blue on postoperative low-back pain and functional outcomes after lumbar open discectomy: a triple-blind, randomized placebo-controlled trial. Journal of neurosurgery Spine. 24:7-15.

Farrokhi MR, Yazdanpanah H, Gholami M, Farrokhi F, Mesbahi AR. 2016. Pain and functional improvement effects of methylene blue injection on the soft tissue around fusion site after traumatic thoracolumbar fixation: A double-blind, randomized placebo-controlled study. Clinical neurology and neurosurgery. 150:6-12.

Feng C, Liu Y, Wang L, Niu D, Han B. 2018. Effects of Early Intracoronary Administration of Nicorandil During Percutaneous Coronary Intervention in Patients With Acute Myocardial Infarction. Heart, lung \& circulation

Fjeldsoe BS, Marshall AL, Miller YD. 2009. Behavior change interventions delivered by mobile telephone short-message service. American journal of preventive medicine. 36:165-73.

Franklin NC, Lavie CJ, Arena RA. 2015. Personal health technology: A new era in cardiovascular disease prevention. Postgraduate medicine. 127:150-8.
Franklin VL, Waller A, Pagliari C, Greene SA. 2006. A randomized controlled trial of Sweet Talk, a text-messaging system to support young people with diabetes. Diabetic medicine : a journal of the British Diabetic Association. 23:1332-8.

Frederix I, Hansen D, Coninx K, Vandervoort P, Vandijck D, Hens N, et al. 2015. Medium-Term Effectiveness of a Comprehensive InternetBased and Patient-Specific Telerehabilitation Program With Text Messaging Support for Cardiac Patients: Randomized Controlled Trial. Journal of medical Internet research. 17:e185.

Furber S, Butler L, Phongsavan P, Mark A, Bauman A. 2010. Randomised controlled trial of a pedometer-based telephone intervention to increase physical activity among cardiac patients not attending cardiac rehabilitation. Patient education and counseling. 80:212-8.

Higgins JP, Thompson SG. 2002. Quantifying heterogeneity in a metaanalysis. Statistics in medicine. 21:1539-58.

Hurling R, Catt M, Boni MD, Fairley BW, Hurst T, Murray P, et al. 2007. Using internet and mobile phone technology to deliver an automated physical activity program: randomized controlled trial. Journal of medical Internet research. 9:e7.

Jadad AR, Moore RA, Carroll D, Jenkinson C, Reynolds DJM, Gavaghan DJ, et al. 1996. Assessing the quality of reports of randomized clinical trials: Is blinding necessary? Controlled Clinical Trials. 17:1-12.

Janssen V, De Gucht V, Dusseldorp E, Maes S. 2013. Lifestyle modification programmes for patients with coronary heart disease: a systematic review and meta-analysis of randomized controlled trials. European journal of preventive cardiology. 20:620-40.

John J, Parikh PB, Thippeswamy G, Kataya A, Loeb C, Tran L, et al. 2018. Sex-related disparities in obstructive coronary artery disease, percutaneous coronary intervention, and mortality in adults with cardiac arrest. International journal of cardiology.

Kang Y, Yang IS. 2013. Cardiac self-efficacy and its predictors in patients with coronary artery diseases. Journal of clinical nursing. 22:2465-73.

Keefer L, Kiebles JL, Taft TH. 2011. The role of self-efficacy in inflammatory bowel disease management: preliminary validation of a diseasespecific measure. Inflammatory bowel diseases. 17:614-20.

Khonsari S, Subramanian P, Chinna K, Latif LA, Ling LW, Gholami O. 2015. Effect of a reminder system using an automated short message service on medication adherence following acute coronary syndrome. European journal of cardiovascular nursing: journal of the Working Group on Cardiovascular Nursing of the European Society of Cardiology. 14:170-9.

Kjaergard LL, Villumsen J, Gluud C. 2001. Reported Methodologic Quality and Discrepancies between Large and Small Randomized Trials in Meta-Analyses. Annals of Internal Medicine. 135:982-9.

Lester RT, Ritvo P, Mills EJ, Kariri A, Karanja S, Chung MH, et al. 2010. Effects of a mobile phone short message service on antiretroviral treatment adherence in Kenya (WelTel Kenya1): a randomised trial. Lancet. 376:1838-45.

Mahonen M, Salomaa V, Keskimaki I, Moltchanov V. 2000. The feasibility of routine mortality and morbidity register data linkage to study the occurrence of acute coronary heart disease events in Finland. The Finnish Cardiovascular Diseases Registers (CVDR) Project. European journal of epidemiology. 16:701-11.

Moher D, Liberati A, Tetzlaff J, Altman DG, Group P. 2009. Preferred reporting items for systematic reviews and meta-analyses: the PRISMA statement. Journal of clinical epidemiology. 62:1006-12. 
Mohr FW, Morice MC, Kappetein AP, Feldman TE, Stahle E, Colombo A, et al. 2013. Coronary artery bypass graft surgery versus percutaneous coronary intervention in patients with three-vessel disease and left main coronary disease: 5-year follow-up of the randomised, clinical SYNTAX trial. Lancet. 381:629-38.

Norman GJ, Kolodziejczyk JK, Adams MA, Patrick K, Marshall SJ. 2013. Fruit and vegetable intake and eating behaviors mediate the effect of a randomized text-message based weight loss program. Preventive medicine. 56:3-7.

Park LG, Howie-Esquivel J, Chung ML, Dracup K. 2014. A text messaging intervention to promote medication adherence for patients with coronary heart disease: a randomized controlled trial. Patient education and counseling. 94:261-8.
Patrick K, Raab F, Adams MA, Dillon L, Zabinski M, Rock CL, et al. 2009. A text message-based intervention for weight loss: randomized controlled trial. Journal of medical Internet research. 11:e1.

Pfaeffli Dale L, Whittaker R, Jiang Y, Stewart R, Rolleston A, Maddison R. 2015. Text Message and Internet Support for Coronary Heart Disease Self-Management: Results From the Text4Heart Randomized Controlled Trial. Journal of medical Internet research. 17:e237.

Sayols-Baixeras S, Lluis-Ganella C, Lucas G, Elosua R. 2014. Pathogenesis of coronary artery disease: focus on genetic risk factors and identification of genetic variants. The application of clinical genetics. 7:15-32.

Wu CJ, Chang AM, Courtney M, Kostner K. 2012. Peer supporters for cardiac patients with diabetes: a randomized controlled trial. International nursing review. 59:345-52. 